\title{
Médecine marchande
}

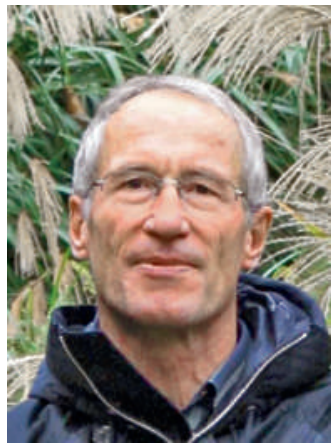

Erhard Taverna
Le philosophe américain Michael J. Sandel est mondialement célèbre pour ses conférences sur la justice. Y a-t-il des limites à la logique de consommation? Pour Sandel, la réponse est oui: «En mettant un prix sur les bonnes choses de la vie, on les avilit.» Nous serions passés d'une économie de marché à une société de marché. Dans son livre «What Money Can't Buy» [1], il explique ce que cela signifie et ouvre ses réflexions par de nombreux exemples. Comme toujours, lorsqu'il est question d'argent, la médecine et le secteur des assurances sont au premier rang.

Ainsi de ces numéros de mobile de certains médecins qui, en échange d'une redevance annuelle, sont joignables en permanence et accordent un rendez-vous dans la journée. Comme personne n'aime attendre, les offres Fast Track fleurissent. Avec, par exemple, les passagers des classes les plus coûteuses dispensés d'attente pour les contrôles de passeports ou d'immigration ou qui bénéficient contre paiement d'une file rapide dédiaée aux heures de pointe. En Chine, il existe un vaste marché noir pour les rendez-vous médicaux dans les hôpitaux publics. Aux Etats-Unis, des groupes de médecins proposent aux particuliers et familles un service exclusif, y compris à domicile et au travail, pour les CEO et autres VIP. Ils réduisent leur patientèle, mais gagnent plus que leurs collègues de la file escargot. L'éthique de la file d'attente vaut bien sûr aussi pour les appels téléphoniques. Puisque les banques, compagnies aériennes et fournisseurs de cartes de crédit mettent des numéros spéciaux à la disposition de leurs meilleurs clients, les médecins font de même.

Lorsque les incitations financières pour de meilleures performances scolaires sont légitimes, les systèmes de gratification des enseignants et les ristournes de vie saine sur les primes le sont aussi. Des piluliers à puce enregistrent la prise du médicament et récompensent comptant l'observance, des adolescentes qui se font vacciner contre le cancer du col de l'utérus reçoivent des bons d'achat et en Angleterre, un programme «pound-for-pound» doit encourager les personnes en surpoids à maigrir. Les assurances vie pour les cadres existent depuis long-temps.

Celles pour les femmes de ménage sont récentes. Une société comme Wal-Mart ou un établissement hospitalier paie les primes de ses employés et encaisse en cas de décès. L'assurance vie, conçue comme un filet de sécurité pour les survivants, devient donc une stratégie financière des entreprises. Le business porte aussi sur les assurances décès des malades incu- rables: un investisseur reprend la police du patient avec un rabais en échange du règlement immédiat et touche le reste au décès. Des hedge funds et des instituts financiers comme le Credit Suisse et la Deutsche Bank ont dépensé des milliards pour racheter les assurances vie de seniors fortunés. En 2007, Goldman Sachs, CS, UBS et d'autres banques ont fondé l'«Institutional Life Markets Association» afin de créer des solutions de marché de capitaux pour le «segment en rapport avec l'espérance de vie et la mortalité». De la spéculation sur la mort cotée en bourse, du même acabit que l'activité de www.stiffs. com, qui propose, contre une mise de départ, de parier sur le décès de célébrités.

On justifie la marchandisation de tous les domaines de la vie par l'efficience soi-disant accrue et la logique propre de l'économie. Le modèle des EtatsUnis fait tôt ou tard école ici. La première assurance complémentaire privée pour les soins ambulatoires a vu le jour au début de l'année. Elle serait tendance, innovante et souhaitée par les clients. Ses souscripteurs sont dispensés de salle d'attente commune et disposent d'en-cas, de journaux et d'Internet dans un espace réservé. Le patient obtient plus vite rendez-vous et choisit lui-même son médecin [2]. Après l'intervention, il bénéficie d'un service de taxi ou d'un stationnement gratuit. Juridiquement, le principe du Fast Track reste délicat, puisque d'après le Tribunal fédéral, les assurances complémentaires ne peuvent prendre en charge que les «véritables prestations complémentaires», qui n'existaient jusqu'ici que dans le domaine stationnaire. Outre des implants et examens plus coûteux, l'assurance couvre des médicaments et méthodes non reconnus en Suisse. Hôpitaux et médecins se réjouissent de ces nouvelles sources de revenus. Idem pour bien des médecins agréés qui ne veulent pas opérer selon le tarif TARMED. Le payeur décide, dit le marché. On peut trouver d'autres idées de profits dans le livre de Michael J. Sandel. Ou se demander comment on veut vivre dans notre société.

Erhard Taverna

\section{Références}

1 Sandel MJ. What Money Can't Buy. Farrar, Straus and Giroux; 2012.

2 Eine Erholungszone für besser Versicherte. Tagesanzeiger du 4 février 2014 